\title{
NOTICE
}

\section{Second Conference of the International Federation of Classification Societies Charlottesville, Virginia June 27-30, 1989}

The International Federation of Classification Societies (IFCS) is organizing its second conference, to be held at the University of Virginia in Charlottesville, from June 27 to June 30,1989 . This conference is devoted to the presentation of theoretical, methadological, and applied papers on classification, pattern recognition, and related methods of statistics and data analysis in the broad sense. It includes mathematical, statistical, and practical investigations in special fields of knowledge, and the interface between classification and the information sciences.

Papers are invited for the meeting. Suitable topics include: Classification, discrimination, aggregation, and clustering methods $\bullet$ Pattern recognition and image analysis methods $\bullet$ Statistical and probabilistic methods for data analysis and classification - Similarity and distance measures, data quality, and reliability • Multidimensional scaling and structure recovery methods $\bullet$ Consensus methods and correspondence analysis methods - Biological taxonomy, genome/molecular sequencing • Analysis and comparison of tree and graph patterns - Artificial intelligence and expert systems for classification - Classification and clustering algorithms and algorithmic aspects • Classification and clustering software for microcomputers and supercomputers • Computer graphics for classificatory problems - Practical applications in fields of biological sciences, information sciences, life sciences, mathematical sciences, medical science, and social sciences.

Please address general inquiries about the conference to IFCS-89, Department of Mathematics, University of Virginia, Charlottesville, VA 22903; (804)924-4919; Bitnet: SJT@VIRGINIA.

If you plan to present a paper, send an abstract in English, no more than 1 page in length, to Robert $F$. Ling, Chairman, IFCS-89 Program Committee, Department of Mathematical Sciences, Clemson University, Clemson, SC 29634-1907, or Bitnet: RFLING@CLEMSON). Submission of an abstract must be accompanied by a title, keywords, name(s) and institutional affiliation(s) of author(s), and the name of the contacting author for papers with multiple authors. The deadline for the submission of abstracts is January 15, 1989. 